\title{
Adventitious reinforcement in free-operant discrimination ${ }^{1}$
}

W. MICHAEL SCHOEL, JENNIFER DAVIS, and M. E. BITTERMAN, UNIVERSITY OF SOUTH FLORIDA, Tampa, Florida 33620

The discriminative performance of rats trained under conditions in which their responses to $S$ - postponed the appearance of $S+$, both for themselves and for yoked-control animals, was found to be superior to the performance of the controls in two rather different experiments. The results demonstrate the pervasiveness of adventitious reinforcement in free-operant discrimination.

When $S-$ is followed directly by $S+$ in the course of discriminative training, the possibility arises that response to $S-$ will be reinforced adventitiously by the appearance of S+ (Dinsmoor, 1950). That discriminative performance under such circumstances may indeed by affected adversely by adventitious reinforcement has been demonstrated recently by Kamil and Davenport (1968). They found that rats whose responses to $S$ - postponed the appearance of $\mathrm{S}+$, both for themselves and for yoked controls, responded less to $S$ than did the controls. No such difference was obtained, however, in a still more recent experiment by Snow and Uhl (1969), nor was a time-out between Sand $\mathrm{S}+$ found to improve the performance of rats trained with fixed S- durations. Snow and Uhl were inclined to attribute the difference in outcome to the fact that the first response to $\mathrm{S}+$ was reinforced in Kamil and Davenport's experiment, while response to $S+$ was reinforced in their own experiment on a VI 15-sec schedule, and they suggested that adventitious reinforcement is a problem only under rather special conditions "in which primary reinforcement is coincident with the change to S+ [p. 220]." We report here two further yoked-control experiments, one like that of Kamil and Davenport and the other with response to $S+$ reinforced on a VI 30-sec schedule. Evidence that discrimination may be impaired by adventitious reinforcement was found in both.

\section{EXPERIMENT 1}

\section{Method}

The Ss of the first experiment, which was much like that of "Kamil and Davenport, were eight albino rats reduced to $85 \%$ of their ad lib weights on a 24 h feeding schedule and trained to press a combination lever and food cup (Bremner \& Trowill, 1962) for 45-mg pellets, first with continuous teinforcement and then on an FR 5 schedule. During this stage of the training, a panel light in the chamber was turned on. After their rates of responding had stabilized, the animals were paired on the basis of the number of responses to $\mathrm{S}-$ in a single session consisting of 4010 -sec presentations of S(panel light on), each followed by $\mathrm{S}+$ (panel light off), the first response to which produced reinforcement and a return to the $\mathrm{S}$ - condition.

In the next eight sessions, one member of each pair was trained with each response in $S$ - resetting the $10-\mathrm{sec}$ timer that scheduled St (O condition), while the other member was trained concurrently as a yoked control ( $C$ condition). The response of the control animal had no effect on the duration of $\mathrm{S}-$-, which was determined entirely by the performance of the experimental animal with which it was yoked, although response to $\mathrm{S}+$ was necessary for reinforcement. In Sessions 10 and 11 , the duration of $\mathrm{S}-$ - was fixed at $10 \mathrm{sec}$ for both members of each pair, and, in the last eight sessions, the contingencies were reversed (that is, the former $C$ animal in each pair became the $O$ animal and the former $O$ animal became the $C$ animal). Each session ended after 40 reinforcements.

\section{Results}

The results of this experiment, which were very much the same from pair to pair, are plotted in Fig. 1 in terms of the mean number of responses to $S-$ in each session. Although the response rates of the two groups were almost identical in the first session, the $O$ animals made many fewer responses than did the yoked animals in Sessions 2-9, when the contingency was in force, and also in Sessions 10-11, when both groups again were tested with fixed S- duration. All animals responded less in Sessions 10-11 than in Session 1, but the decline in level of responding was greater in each $O$ animal than in its control. In Session 12 the number of responses made by both groups increased, because the length of the session increased (the session length for each pair now was determined by the behavior of the former $\mathrm{C}$ animal). In subsequent sessions the number of responses made by both groups declined, but more rapidly and to a lower level in each new $O$ animal than in its control.

\section{EXPERIMENT 2}

\section{Method}

The Ss of the second experiment, which was pattemed after that of Snow and Uhl
(1969), were 10 hooded rats reduced to $85 \%$ of their ad lib weights on a $24-\mathrm{h}$ feeding schedule and trained to leverpress for $45-\mathrm{mg}$ pellets on a VI $30-\mathrm{sec}$ schedule. Each chamber contained a single centered lever, with a food cup below and to the left of the lever. A houselight, which was turned on at the start of each session and turned off at the end of the session, dimmed perceptibly as each reinforcement was delivered. The training was carried out both in the presence and in the absence of an auditory stimulus of moderate intensity, consisting of a $220-\mathrm{Hz}$ train of $0.2-\mathrm{msec}$ pulses. After responding had stabilized, the animals were paired on the basis of rate to form two groups, $O$ and $C$, and trained in a discrimination between the presence and absence of the auditory stimulus, which was programmed as follows: In each session there were five 2-min presentations of St (the presence of the sound was positive for three pairs of animals and its absence positive for the other two pairs) during which response was reinforced on a VI $30-\mathrm{sec}$ schedule. Alternated regularly with these $S+$ presentations were unreinforced $S-$ presentations (half of the sessions began with $\mathrm{S}+$ and half with $\mathrm{S}-$-) whose minimal duration was $2 \mathrm{~min}$. In Sessions 1-7 and 9-11, any response of an $\mathrm{O}$ animal in the last $30 \mathrm{sec}$ of an $\mathrm{S}-$ presentation postponed the termination of $\mathrm{S}-$ for $30 \mathrm{sec}$, both for itself and for the C animal yoked with it. In Sessions 8 and 12, the duration of $\mathrm{S}$ - was fixed at $2 \mathrm{~min}$ for all animals.

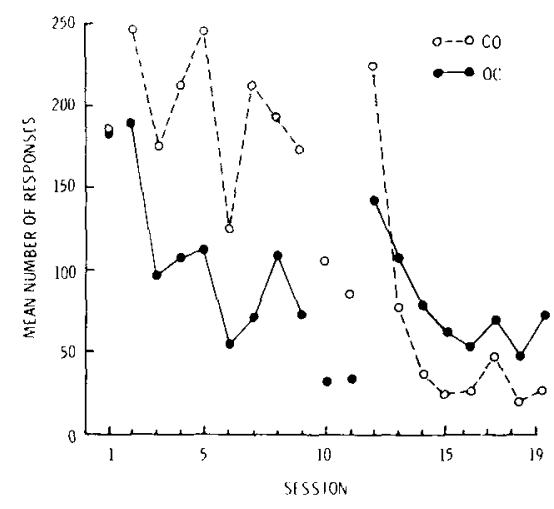

Fig. 1. Mean number of responses to $\mathrm{S}-$ in each session of Experiment 1 made by animals trained with $S_{+}$postponement in Sessions 2-9 and as yoked controls in Sessions 12-19 (OC), and by animals trained under the two conditions in the reverse order (CO). The unconnected points are for sessions in which the duration of $\mathrm{S}-$ was constant for all animals. 

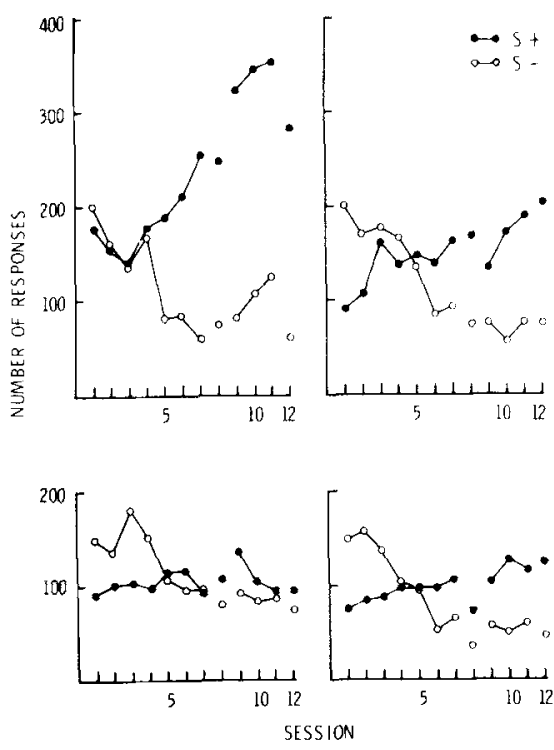

Fig. 2. Number of responses to $S+$ and $S-$ made by two pairs of animals in Experiment 2. The upper plots are for animals trained with $S+$ postponement, and the lower plots are for their yoked controls. The unconnected points are for sessions in which the duration of $\mathbf{S}$ - was constant for all animals.

\section{Results}

The results of the experiment are rather clear. All of the $O$ animals responded differentially to the two stimuli, and the degree of differentiation was greater in each $\mathrm{O}$ animal than in its yoked control. Only one of the $C$ animals, in fact, gave any reliable evidence of discrimination. In Fig. 2 the performance of two of the pairs is plotted in terms of the number of responses to each stimulus in each session. The plots in the upper portion are for $\mathrm{O}$ animals and those in the lower portion are for their yoked controls. The first $O$ animal, for which the presence of the auditory stimulus was $S^{+}$, was one of the two best, but its control gave no reliable evidence of discrimination. The performance of the second $\mathrm{O}$ animal, for which the auditory stimulus was negative, was poorer than that of any other $O$ animal, but it discriminated sharply in the later sessions, its highest rate of response to any $S$ being lower than its lowest rate of response to any St. By contrast, its yoked control, whose performance was better than that of any other $\mathrm{C}$ animal, continued on a number of occasions to respond more rapidly to $S-$ than to $S+$.

\section{DISCUSSION}

The results of the first experiment are in accord with those of Kamil and Davenport (1968). Together with the results of the second experiment, they indicate that the effect of adventitious reinforcement may be more pervasive than Snow and Uhl (1969) have suggested and that steps to guard against it might well be taken routinely in discriminative training. Of the several methods available for dealing with the difficulty, the most effective perhaps is St postponement, the very method that has been used to demonstrate it.

\section{REFERENCES}

BREMNER, F. J., \& TROWILL, J. A. A combined manipulandum-reinforcement arrangement Joumal of the Experimental Analysis of Behavior, 1962, 5, 339-340.

DINSMOOR, J. A. A quantitative comparison of the discriminative and reinforcing functions of a stimulus. Journal of Experimental Psychology, 1950, 40, 458-472.

KAMIL, A. C., \& DAVENPORT, J. W. The role of adventitious reinforcement in operant discrimination. Journal of Experimental Psychology, 1968, 76, 609-617.

SNOW, M. E., \& UHL, C. N. Effects of time out and $S+$ postponement training procedures on free-operant discrimination acquisition. Psychonomic Science, 1969, 14, 218-220.

\section{NOTE}

1. This research was supported by Grant MH 17736 from the National Institute of Mental Health. 